Hypothesen, die mit elektrischen Vorgängen, Elektronen u. dergl, arbeiten.

Nun hatte der Verfasser in einer gemeinsamen Arbeit mit R. Ed. Li esegan.g ${ }^{27}$ ) unierschieden zwischen der physikalischen oder Kornoberflächenentwicklung und der chemischen oder Korntiefenentwicklung, und man wird unserer Auffassung beipflichten, dab bei chemischer Entwicklung das große Korn deshalb mehr leisten kann als das kleinere, weil; selbst gleiche wirksame Keimmenge angenommèn, im ersteren Falle mehr Sílber pro Korn reduziert werden kann als im zweiten. Bei der Kornoberflächenentwicklumg liegen aber offenbar die Verhältnisse anders, denn hier kommt es vor allem auf die Anzahl der Keime an der. Gesamtoberfläche der Körner an; da diese bei der physikalischen Entwicklung ausschlieblich in Frage kommt. Wenn ich nun auch in bezug auf die Einzelheiten unserer Auffassung anf die erwăhinte Ârbeit zurückverweise, șo möge hier doch noch eine Ergänzung nachgetragen werden.

Der Unterschied in der Lichtempfindlichkeit der verschiedenen Bromsilbergelatineemulsionen, einerseits der höchstdispersen sogen. kornlosen Emulsionen, wie sie für das Lippmann'sche Interferenz-Parbenverfahren benutzt werden, andererseits der modernen höchstempfindlichen Porträt- und Momentplatten, kann auf mindestens 1:40000 angenommen werden. Von dieser Steigerung ist nur ein Teil, wenn auch der größte, wohl auf Kosten der Vergrőßerung des Kornes und damit verbundener innerer Vorgänge zu setzen, vielleicht durchschnittlich die Erhöhung der Sensibilität auf das 1000 fache. Der letzte Grad der Empfindlichkeitszunahme ist indessen hauptsächlich den Reduktionsspuren zuzuschreiben, die sich bei der Reifung infolge der Wirkung der meist ammoniàkalischen Gelatinelösung auf das Bromsilber

27) Koll,-Zeitschr. 9, 290 (1911); Ede r's Jahtb. $1912,18$. bilden ${ }^{28}$ ). Entfernt man durch geeignete Silberlösungsmittel einerseits aus einer sehr hochempfindlicher, andererseits aus einer mittelkörnigen Diapositivplatte die Reduktionskeime, so verschwindet der vorher vorhandene Unterschied in der Empfindlichkeit zum weitaus größten Teil, weil die Ueberlegenbeit der hochempfindlichen Platte hier fast nur noch auf dem Vorhandensein der Reduktionskeime beruhte. Die Wirkung der Reduktionskeime hat man sich nun wohl so vorzustellen, dab sie bei der Belichtung katalytisch die Zersetzung des Bromsilbers an diesen Punkten beschleunigen. Diese Wirkung wird von der äuBersten Kornoberfläche, wo die Reduktionskeime sich be: finden, nach dem Korninnern zu fortschreiten. Von dieser für die weitere Reduktion des Kornes günstigen Veränderung wird aber hauptsächlich nur die Korntiefenentwicklung Vorteile ziehen könren, während die physikalische Entwicklung weniger davon berührt werden wird.

Man wird danach die oben angedeutete Schwierigkeit, auch bei der chemischen Entwicklung ausschlieblich eine Keimwirkumg des latenten Bildes anzunehmen, mcht mehr für unüberwindlich halten, wenn auch zugegeben werden muB, dak in den angedeuteten Fragen - noch manche Einzelheiten geklärt werden müssen, die allerdings teilweise experimentell schwer zugänǵlich sind. Andererseits aber würde die Annahme besonderer Vorgänge bei der Belichtung gerade nur der hochempfindlichen Schichten eine Diskontinuität darstellen, die zuzugeben erst dann notwendig wäre, wenn man für jene anderen Vorgänge experimentelle. Beweisè hätte, die bislang nicht geliefert worden sind.

Vorläufig scheint mir die Entwicklung des latenten Bildes, in allen Fällen ein genügend zuverlässiges Reagens auf vorhandene Silberkeime darzustellen.

Wissenschaftl. Laboratorium der Trockenplattenfabrik Kranseder \& Co. in München.

28) L ü p po-Cram er, Photogr. Mitteil. 1909, 328.

\title{
Ueber die Reduktion des Natriumsilberthiosulfats mit Hydrosulfit, II.
}

Von Albert Steigmann (Heilbronn).

(Eingegangen am 29. Sept, 1920.)

Es wurde darauf hingewiesen, dab Hydrosulfit mehr als alle anderen photographischen Entwickler die Fähigkeit besitzt, freiwillig zahlreiche reduktionsauslösende Keime bei der Reduktion von Sịlbersalzen zu. bilden. Eine Erklärung dafür wurde noch nicht versucht.
Nach P. P, von We imàrn hängt die Zahl der Punkte, an denen bei der freiwilligen Entstehung der beständigen, festen Phase die Kristallisation beginnt, vom Uebersättigungsgrade der Lobsung - in unserem Falle von der Uebersättigung an Silber - ab. 
Je größer die Uebersättigung an Silber ist, desto mehr Keine müssen nach den Ueberlegungen von Weimarn entstehen, um so kleiner werden die Kriställchen sein, de sich im Verlauf der Reduktion bilden.

Man könnte versucht sein, die starke Uebersättigung von Silberbösungen, wie man sie bei Reduktion von Hydrosulfit erhäl, auf eine groke Reduktionsgeschwindigkeit zurückzuführen. Man erhält aber auch bei langsamer Reduktion von Hydrosulfit z. B. bei Reduktion von Natrium. silberthiosulfat mit großem Thiosulfatüberschub gelbe bzw. gelbbraune Sole, was auf zahlreiche Keime bzw. starke Uebersättigungen schliehen 1ät. Umgekehrt resultieren bei rascher Reduktion von gelatinehaltigen Silbersalzlösungen mit anderen Entwicklern blaue Sole.

Es ist anzunehmen, daß es in der Natur des Hydrosulfits liegt, zahlreiche Keime zu bilden und daB die starken Uebersättigurngen, welche bei Reduktion mit Hydrosulfit auftreten, vielleicht auf dessen hohes Reduktionspotential zurückzufühitren sind.

Man kaun jedoch die Reduktion mit Hydro. sulfit auch so leiten, daB weniger starke Uebersättigungen auftreten, indem man beispielsweise die Reduktionsgemische mit Keimen impft oder innerhalb derselben leicht reduzierbare Silbersalze entstehen laBt, z. B. Jodsilber innerhalb des durch ThiosulfatüberschuB schwer reduzierbar gewordenen Natriumsllberthiosulfats.

In diesem Falle entstehen aus dem Jodsiber Silberkeime bzw. Keimkomplexe, bevor aus dem schwer reduzierbaren Natriumsilberthiosulfat mit Thiosulfatüberschubs solche entstehen können. Man erreicht dabei genau dasselbe wie bei $\mathrm{Zu}$ gabe von Keimen aus einer Keimlösung, -Die aus $\mathrm{Ag} J$ entstandenen Keitne, welche wie Impfkristalle wirken, lassen es im weiteren Verlauf der Reduktion zu keiner Uebersättigung mehr kommen, durch die neue Kristallisationszentren entstehen könuten.

Natürlich werden sich bei der Reduktion durch lokale Uebersättigungen immer wieder vorübergehend Amikronen bilden, die jedoch nuir in bezug auf stark übersättigte lösungen beständig sind. Da aber übersättigte Lösungen wegen der vorhandenen aus Agl entstandenen Keime, die sofort jede Uebersättigung aufheben, nicht mehr entstehen, so lösen sich die Amikronen zugunsten der primär enistandenen Keime auf. Man hat sich jedoch die Amikronen vón verschicdener Gröberiordnung $2 w$ denken. Die gröberen unter ihnen, die im Lauf der Reduk. tion trolz der primären Keine durch gröbere lokale Inhomogenitäten entstehen, werden nicht gelöst, sondern betäligen sich ebenfalls als Keime, die den primär entstandenen Silber wegnèhmen und sie dadurch an weiteren Wachstum verhindern. Erhöht man die Löslichkeit dęs Silbers durch schwache Säuren, so werden auch die gröberen Amikronen zugunsten der primären Keime gelöst. Dieser Vorgang, der sich beim Wachstum der gelben Silberteilchen zu roten und blauen abspielen dürfte, wurde bereits beschrieben wnd nach Läpo-Cramert als Ostwaldreifung des kolloiden Silbers bezeichnet. -. Die Ostwaldreifung des Silbers bleibt aus, wenn man das Bisulfit des zerfallenden Hydrosulfits durch Soda abstumpft. Die vorübergehend durch lokale Uebersättigung des Reduktionsgemisches entstandenen gröberen Amikronen werden dann nicht mehr gelöst, sondern schlagen itm weiteren Vetlauf der Reduktion ebenfalls Silber auf sich nieder, das sie den anderen Keimen wegnehmen. - Die Ergebnisse der bisherigen Untersuchungen, welche angestellt wurden, um die günstigsten Bedingungen für ein neues Verfahren der Entwicklung nach dem Fixieren zu findon, gestatteten dasselbe so auszuarbeiten, dab die bestmöglichen Resultate erzielt wurden. Es war erwünscht, das Verfahren so zu gestalten, dab sich damit möglichst rasch und billig entwickeln ließ3. Da man bei physikalischer Entwicklung verhältnismäBig viel Silber für die Entwicklerlösung braucht, muBte nach einer billigen Siberquelle gesucht werden, welche sich denn auch in verbrauchten Fixierbädern finden ließ. Dieselben fallen bei jedem Photographen ab und körinen auf das vier-bis fünffache nit Thiosulfatlösung $1: 5$ verdünnt werden.

Größere Schwierigkeiten waren zu üherwinden, als es galt, die Entwicklung möglichst rasch durchzuführen, ohne dabei viel Silber in Form von Schlarmm zu verlieren. Das Silber (Siberschlamm) fällt in der Fntwicklerlösung nur dann aus, wenn dieselbe an Silber stark übersättigt ist. Es ist destralb zur Vermeidung von Silberverlusten dafür zu sorgen, daB die Uebersältigung ständig aufgehoben und möglichst viel Silber in der Zeireinheit zur Deckung des Bildes verbraucht wird, wodurcl natïrlich auch eine beschleunigte Entwickelung einsetzt. Man erreicht dies am besten dadurch, dab man mit dem latenten fixierten Bild zahlreiche Belichtungssilberkeime in die Entwicklerlösung bringt tund aus bisulfitsauren,

1) Lüppo-Cramer, loc.cit. 
jodkäliumhaltigen Lösungen reduziert, in denen sich, wie wir gesehen laben, die spontan in der Entwicklerlosung entstehenden Amikronen zugunsten der schon vorhandenen - in diesem Falle der Belichtungssilberkeime des latenten Bildes - ..- auflösen.

$\mathrm{Da}$ auf wenig gereifter Chlor- und Chlorbromsilberschichten bei genügend langer $\mathrm{Bc}-$ lichtung sehr viele Keime entstehen, wie aus Untersurhungen von Lüppo-Cramer ${ }^{2}$ ) hervorgeht, sind dieselben für die Entwickelung mit Hydrosulfit am besten geeignet. Die einzelnen Belichtungskeime werden, da sie in grober Zahl vorhanden sind, nicht stark wachsen. Die Deckung des Bildes geschieht in diesem Falle nicht, wie bei der chemischen Entwicklung gereifter Emulsionen, durch verhältrismäßig wenige grobe Silberkörner, sondern durch eine Unzahl kleiner Silberteilchen. Da das Silber der nach dem neuen Verfahren entwickelten Gaslichtpapiere kolloiddispers ist, lassen sich die Bilder leicht nach den verschiedensten Tonungsverfahren tonen. Jodkalium leistet bei der Entwickelung von Gaslichtpapieren gute Dienste zur Beschleunigung der Entwickelung. Soda, welches bekanntlich die Reduktion des Natriumsilberthiosulfats ebenfalls beschleunigt, darf nicht verwendet werden, da es das Bisulfit abstumptt, welches, wie wir gesehen haben, entstehende Amikronen zugunsten der Belichtungskeime löst. Die amikrospischen Silberteilchen bleiben in alkalischer Lösung erhaiten und es entsteht dadurch ein Gelbschleier, der den Belichtungskeimen alles Silber wegnimmt und das Bild überdeckt. Das gilt hauptsächlich für die Entwickelung gereifter Emulsionen, auf denen immer verhältnismäBig wenig Belichtungskeime entstehen, so daß in der Entwicklerlösung (die wenigen Keirne schlagen nur geringe Mengen Silber auf sich nieder) starke Uebersättigungen eintreten, was zur Bildung zahlreicher Schleierkeime führt.

Wie wir schon oben gesehen haben, kann das Silber, das bei dem neuen Verfahren nötig ist, direkt. aws dem Bad, in dem die Bilder fixiert werden bzw. aus verbrauchten Fixierbädern bezogen werden, dank der Fähigkeit des Hydrosulfits Natriumsilberihiosulfat selbst bei großem Thiosulfatäberschuß zu reduzieren.

2) Lü po-Cran in r, Plotographische Korrespondenz $1913,509$.
Die wenig gereiften, uberexponierten, hartarbeitenden Gaslichtpapiere, die nach dem nenen Verfahren entwickelt werden sollen, kommen in ein verbrauchtes Fixierbad, das vorteilhaft mit frischer Fixiernatronlösung verdünnt wird. Nach zwei Minuten ist das Bild schon genügend ausfixiert, um es am. Tageslicht mit Hydrosulfit aus dem Fixierbad heraus eventl. unter Zusatz von Jodkalium entwickeln zu können. Das Bild, das in nassem -Zustande gelbe Farbe zeigt, wird beim Auftrocknen dunkelbraun.

Bei Entwicklung mit Natriumbydrosulfit er* reichen die einzelnen Silberteilchen eine maximale Gröbe von $0,03 \mu$. Man kann sich deuken, daß deshalb zur Deckung von Bildern, die mit Hydrosulfit entwickelt werden; bedeutend mehr Silberteilchen nötig sind, als für die gewöhnliche Entwicklung, bei der das Silberkorn verhältnismäfig grob ist. Es sind deshalb für das neue Verfahren auch aus dieserm Grunde (also nicht allein zur Kürzung der Entwicklungszeit und zur Vermeidung von Silberverlusten) nur solche Halogensilberemulsionen zu gebrauchen, auf denen bei genügend langer Belichtung zahlreiche Silberkeime entsteben können, die dann durch einen der Kristallisation ähnlichen Prozeß $z u$ gelben und braunen Sitberteilichen heranwachsen und durch ihre große Zahl zur Deckung des Bildes führen. $Z$ u diesen Emulsionen gehören die bereits erwähnten, wenig gereiften, stark chlorsilberhaltigen Schichten von Gaslichtpapieren. Völlig unbrauchbar sind gereifte Emulsionen, die für Momentaufnahmen in Verwendung sind.

Das neure Verfahren ist hauptsächlich insofern von Interesse, als es erlaubt, einen genauen Einblick in den Mechanismus der physikalischen Entwicklung zu gewinnen, der sich von dem der gewöhnlichen Entwickiung - wie-schon Lü p po-Cramer feststellte ${ }^{3}$ ) grundsätzlich unterscheidet.

Von dem bekannten Verfahren zur Entwicklung nach dem Fixieren dürfte das neue woht das feinste bildgebende Silber liefern, womit sich auch die Gradationsabweichungen gegenüber der gewönlichen Entwicklung und anderen Verfahren der physikalischen Entwicklung erklären lassen.

3) Lijppo-Cramer, Atelier des Photographen 1908,1 